\title{
Optimization of apodized pupil Lyot coronagraph for ELTs
}

\author{
P. Martinez ${ }^{1}$, A. Boccaletti ${ }^{1}$, M. Kasper ${ }^{2}$, P. Baudoz $^{1}$, and C. Cavarroc ${ }^{1}$ \\ 1 LESIA, Observatoire de Paris Meudon, 5 pl. J. Janssen, 92195 Meudon, France \\ e-mail: patrice.martinez@obspm.fr \\ 2 European Southern Observatory, Karl-Schwarzschild-Strasse 2, 85748 Garching, Germany
}

Reveived 24 April 2007 / Accepted 14 July 2007

\section{ABSTRACT}

\begin{abstract}
Aims. We study the optimization of the Apodized Pupil Lyot Coronagraph (APLC) in the context of exoplanet imaging with groundbased telescopes. The APLC combines an apodization in the pupil plane with a small Lyot mask in the focal plane of the instrument. It has been intensively studied in the literature from a theoretical point of view, and prototypes are currently being manufactured for several projects. This analysis is focused on the case of Extremely Large Telescopes (ELTs), but is also relevant for other telescope designs.

Methods. We define a criterion to optimize the APLC with respect to telescope characteristics such as central obscuration, pupil shape, low-order segment aberrations and reflectivity as functions of the APLC apodizer function and mask diameter. Specifically, the method was applied to two possible designs of the future European-ELT (E-ELT).

Results. Optimum configurations of the APLC were derived for different telescope characteristics. We show that the optimum configuration is a stronger function of central obscuration size than of other telescope parameters. We also show that APLC performance is quite insensitive to the central obscuration ratio when the APLC is operated in its optimum configuration, and demonstrate that APLC optimization based on throughput alone is not appropriate.
\end{abstract}

Key words. techniques: high angular resolution - instrumentation: high angular resolution - telescopes

\section{Introduction}

Over the past ten years many diffraction suppression systems have been developed for direct detection of extrasolar planets. At the same time, promising ground-based projects were proposed and are currently under development like SPHERE at the VLT (Beuzit et al. 2006a) and GPI (Macintosh et al. 2006). Larger telescopes are desirable to improve performance of exoplanet searches towards lower masses and closer angular distances, ideally down to Earth-like planets. Several concepts of Extremely Large Telescopes (ELTs) are currently being studied worldwide: European-ELT (E-ELT, Dierickx et al. 2004), Thirty Meter Telescope (TMT, Nelson \& Sanders 2006), Giant Magellan Telescope (GMT, Johns et al. 2004).

The characteristics of these telescope designs may have an impact on their high contrast imaging capabilities. Parameters such as central obscuration, primary mirror segmentation, and large spider arms, can impose strong limitations for many coronagraphs. It is therefore essential to indentify and evaluate a coronagraph concept which is well-suited to ELTs.

The Apodized Pupil Lyot Coronagraph (APLC) is one of the most promising concepts for ELTs. Its sensitivity to central obscuration is less critical than, e.g., for phase masks (Rouan et al. 2000; Mawet et al. 2005) but the APLC still allows for a small inner working angle (IWA) and high throughput if properly optimized. Other amplitude concepts (e.g. Kuchner \& Traub 2002) are also usable with centrally obscured aperture but suffer from low throughput especially if the IWA is small. The potential of the APLC has been demonstrated for arbitrary apertures (Aime et al. 2002; Soummer et al. 2003) and specific solutions for obscured apertures have been proposed (Soummer 2005).
In this paper, we analyze the optimization of the APLC and evaluate its sensitivity with respect to the main parameters mentioned above. In Sect. 2 we briefly revise the APLC formalism and define a criterion for optimizing the coronagraph parameters. The impact of several telescope parameters on the optimal configuration is evaluated in Sect. 3. Section 4 shows an application of the APLC optimization to two potential ELT designs. We then derive conclusions.

\section{Apodization for centrally obscured pupils}

\subsection{Formalism}

In this section, we briefly revise the formalism of the APLC. The APLC is a combination of a classical Lyot coronagraph (hard-edged occulting focal plane mask, hereafter FPM) with an apodization in the entrance aperture.

In the following, for the sake of clarity, we omit the spatial coordinates $r$ and $\rho$ (for the pupil plane and focal plane respectively). The function that describes the mask is noted $M$ (equal to 1 inside the coronagraphic mask and to 0 outside). With the mask absorption $\varepsilon$ ( $\varepsilon=1$ for an opaque mask), the FPM is then equal to:

$1-\varepsilon M$

$P$ is the telescope aperture, and $\phi$ the profile of the apodizer. $\Pi$ describes the pupil stop function, which is considered - in the initial approximation - to be equal to the telescope aperture $(\Pi=P)$. The coronagraphic process, corresponding to propagation from the telescope entrance aperture to the detector plane, is expressed in Eqs. (2) to (6). Planes A, B, C and D correspond to the telescope aperture, the coronagraphic focal plane, 

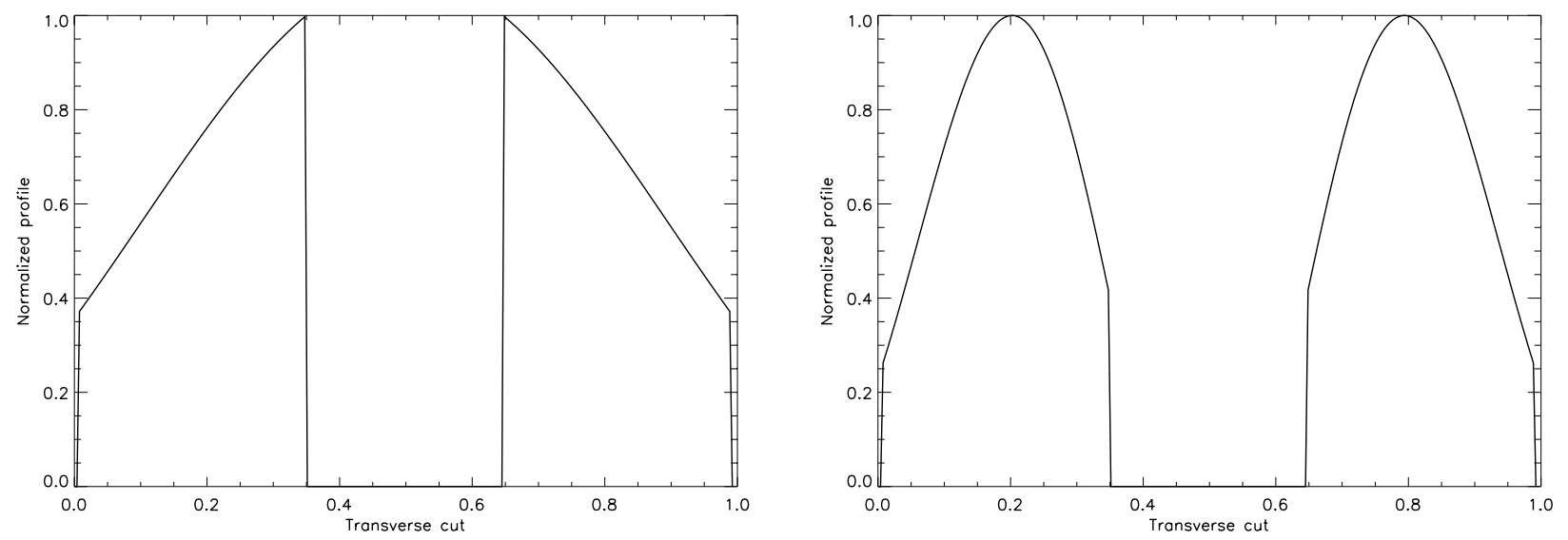

Fig. 1. Typical apodizer shape for the bell regime (left) and the bagel regime (right). Central obscuration is $30 \%$.

the pupil stop plane and the detector plane respectively as defined in Fig. 2. The Fourier transform of a function $f$ is noted $\hat{f}$. The symbol $\otimes$ denotes the convolution product. The entrance pupil is apodized in the pupil plane:

$\psi_{\mathrm{A}}=P \phi$.

The complex amplitude of the star is spatially filtered (low frequencies) by the FPM:

$\psi_{\mathrm{B}}=\hat{\psi}_{\mathrm{A}} \times[1-\varepsilon M]$.

The exit pupil image is spatially filtered (high frequencies) by the stop:

$\psi_{\mathrm{C}}=\hat{\psi}_{\mathrm{B}} \times \Pi$

$\psi_{\mathrm{C}}=\left[\psi_{\mathrm{A}}-\varepsilon \psi_{\mathrm{A}} \otimes \hat{M}\right] \times \Pi$.

The coronagraphic amplitude on the detector plane becomes:

$\psi_{\mathrm{D}}=\hat{\psi}_{\mathrm{C}}=\left[\hat{\psi}_{\mathrm{A}}-\varepsilon \hat{\psi}_{\mathrm{A}} M\right] \otimes \hat{\Pi}$.

The coronagraphic process can be understood as a destructive interference between two waves (Eq. (5)): the entrance pupil wave $P \phi$, noted $\psi_{\mathrm{A}}$ and the diffracted wave by the mask (corresponding to $\left.\varepsilon \psi_{\mathrm{A}} \otimes \hat{M}\right)$. In the non-apodized case $(\phi=1)$, the two wavefronts do not match each other, and the subtraction does not lead to an optimal starlight cancellation in the Lyot stop pupil plane. A perfect solution is obtained if the two wavefronts are identical (i.e., the diffracted wave by the mask $(M)$ is equal to the pupil wave in amplitude). This latter case is obtained with the Apodized Pupil Phase Mask Coronagraph (Roddier \& Roddier 1997; Aime et al. 2002; Soummer et al. 2003). For the APLC, the coronagraphic amplitude is minimized and proportional to the apodizer function.

Considering a pupil geometry, the apodization function is related to the size of the FPM. More precisely, the shape of the apodizer depends on the ratio between the extent of $\hat{M}$ and the central obscuration size (Soummer 2005; Soummer et al. 2007). If the extent of $\hat{M}$ is bigger than the central obscuration, the apodizer takes a "bell" shape (typically it maximizes the transmission near the central obscuration of the pupil (Fig. 1, left). On the contrary, if the extent of $\hat{M}$ is smaller than the central obscuration, the apodizer takes a "bagel" shape reducing transmission in the inner and outer part of the pupil (Fig. 1, right). Thus, the apodizer shape depends on both the FPM size and the central obscuration size.

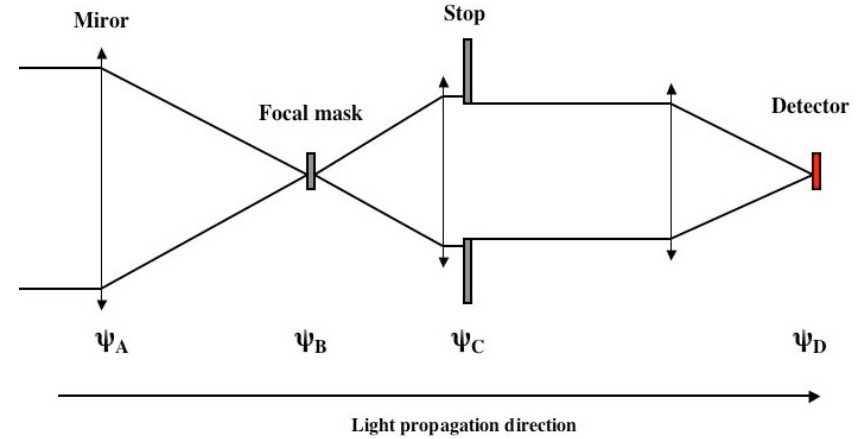

Fig. 2. Diagram of a coronagraph showing the pupil plane containing the apodizer $\left(\psi_{\mathrm{A}}\right)$, the focal plane with the FPM $\left(\psi_{\mathrm{B}}\right)$, the pupil image spatially filtered by the stop $\left(\psi_{\mathrm{C}}\right)$ and the detector plane $\left(\psi_{\mathrm{D}}\right)$.

Throughputs (apodizer transmission/pupil transmission) as a function of the FPM size is given in Fig. 3 for different obscuration sizes ( 15 to $35 \%$ ). These curves show a second maximum corresponding to the transition between the two apodizer regimes which depends on the central obscuration size. Since apodizer throughput does not evolve linearly with FPM diameter, it is not trivial to determine the optimal FPM/apodizer combination. Moreover, throughput might not be the only relevant parameter when optimizing a coronagraph.

A thorough signal-to-noise ratio analysis is definitely the right way to define the optimal FPM/apodizer system, but this would be too instrument-specific for the scope of this study. Here, we investigate a general case for any telescope geometry and derive the corresponding optimal FPM size.

\subsection{APLC optimization criteria}

Usually, in Lyot coronagraphy, the larger the FPM diameter the larger the contrast. However, in the particular case of the apodized Lyot coronagraph the transmission of an off-axis pointlike object is not linear (Fig. 3) and a trade-off has to be made between contrast and throughput. This problem has been studied by Boccaletti (2004) who evaluated optimal Lyot stops for any telescope pupil geometry and for any type of coronagraph. Based on this study, we propose a criterion adapted to the APLC to optimize the apodizer/ FPM combination. This criterion maximizes the coronagraphic performance while minimizing the loss of flux of the off-axis object. While not replacing a thorough signal-to-noise ratio evaluation, our criterion takes into account 


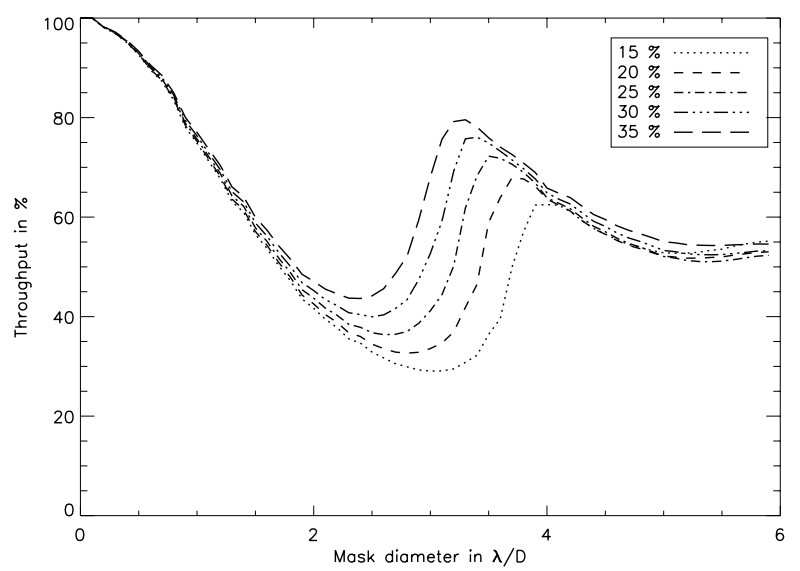

Fig. 3. Apodizer throughput (relative to full transmission of the telescope pupil) as a function of FPM diameter for different obscuration sizes.

the modification of the off-axis PSF (in intensity and in shape) when changing the coronagraph parameters.

Several metrics can be used to quantify the capability of a coronagraph (e.g. Boccaletti 2004). Here, we use the contrast $(\mathscr{C})$ averaged over a range of angular radii:

$\mathscr{C}=\frac{\max \left(\left|\psi_{\mathrm{D}}(\rho, \alpha)_{\varepsilon=0}\right|^{2}\right)}{\left(\int_{0}^{2 \pi} \int_{\rho_{\mathrm{i}}}^{\rho_{\mathrm{f}}}\left|\psi_{\mathrm{D}}(\rho, \alpha)\right|^{2} \rho \mathrm{d} \rho \mathrm{d} \alpha\right) / \pi\left(\rho_{\mathrm{f}}{ }^{2}-\rho_{\mathrm{i}}{ }^{2}\right)}$

where $\mathscr{C}$ is expressed in polar coordinates $\rho$ and $\alpha$. We denote by $\rho_{\mathrm{i}}$ and $\rho_{\mathrm{f}}$ the short radii and the large radii, respectively, defining the area of calculation for $\mathscr{C}$.

The attenuation of the off-axis object is given by the ratio of maximum image intensity with the apodizer only to that without the coronagraph, i.e., without the apodizer and the FPM. This quantity differs from the throughput, since it also takes into account the modification of the PSF structure when changing the apodizer profile:

$\max \left(\frac{\left|\psi_{\mathrm{D}}(\rho, \alpha)_{\varepsilon=0}\right|^{2}}{|\hat{P}(\rho, \alpha)|^{2}}\right)$.

Now, let us define the criterion $C_{\mathscr{C}}$ as the product of $\mathscr{C}$ and Eq. (8).

$C_{\mathscr{C}}=\mathscr{C} \times \max \left(\frac{\left|\psi_{\mathrm{D}}(\rho, \alpha)_{\varepsilon=0}\right|^{2}}{|\hat{P}(\rho, \alpha)|^{2}}\right)$.

The first term of $C_{\mathscr{C}}$ (Eq. (7), which characterizes the performances of the coronagraphic system) is then adapted to the region of interest in the coronagraphic image and can be well matched to the instrument parameters.

The second term (Eq. (8)) takes into account the modification of the PSF structure when changing the apodizer profile and guarantees a reasonably moderate attenuation of the off-axis PSF maximum intensity (i.e., guarantees that when the coronagraph rejects the star it does not reject the planet as well).

Although our criterion cannot replace a thorough signal-tonoise ratio analysis (no instrumental model, no noise terms), it presents a reasonable approach by assuming the residual light leaking through the coronagraph as noise. Our criterion allows us to investigate the trade-off between performance and throughput while keeping the study general and independent of a specific instrument setup.
Moreover, the validity of this criterion is supported by the pupil stop optimization study of Boccaletti (2004) who facied a problem similar to ours, and also by the results presented and discussed in this paper.

\section{Sensitivity analysis}

\subsection{Assumptions}

Based on the previously defined criterion, we now analyze the behavior of several telescope parameters as a function of the size of the FPM (and hence APLC characteristics) with the main objective of exploring possibilities of how to optimize the APLC configuration for a given ELT design. One advantage of $C_{\mathscr{C}}$ is that the area of optimization in the focal plane can be well matched to the instrumental parameters. For this reason, we have limited the search area and investigated $C_{\mathscr{C}}$ only between $\rho_{\mathrm{i}}=3 \lambda / D$ at small radii and $\rho_{\mathrm{f}}=100 \lambda / D$ at large radii. These limits correspond to the IWA (distance at which an offaxis object reaches a significant transmission) and to the highorder Adaptive Optics (AO) cut-off frequency, respectively. At radii larger than the AO cut-off frequency, the coronagraph will only have a minor effect since atmospheric turbulence is not corrected and atmospheric speckles dominate.

For the simulations presented in the next sections, we assume a circular pupil with $30 \%$ central obscuration. The central obscuration ratio is left as a free parameter only in Sect. 3.2.1 where we evaluate its impact. The pupil stop is assumed identical to the entrance pupil including spider arms (Sivaramakrishnan \& Lloyd 2005). Section 3.2.2, where the impact of the spider arms' size is analyzed, assumes 42-m telescope. Elsewhere, simulation results do not depend on the telescope diameter. Apodizer profiles were calculated numerically with a Gerchberg-Saxton iterative algorithm (Gerchberg \& Saxton 1972). The pixel sampling in the focal plane is $0.1 \lambda / D$, and the pupil is sampled with 410 pixels in diameter. When phase aberrations are considered we adopt a wavelength of $1.6 \mu \mathrm{m}$ corresponding to the $H$-band in the near infrared.

\subsection{Critical parameter impacts}

In the following sub-sections, we study the impact of two major categories of diffraction effects. The first category deals with amplitude variations: central obscuration, spider arms, primary mirror segmentation, segment-to-segment reflectivity variation, and pupil shear (misalignment of the coronagraph stop with respect to the instrument pupil). Inter-segment gaps and other mechanical secondary supports are not considered, since they would require finer pixel sampling in the pupil image, resulting in prohibitively large computation times with a non-parallel computer. In addition, some mechanical secondary supports can be much smaller than the main spider arms. At the first approximation, their effects can be considered to be similar to those produced by spider arms.

The second category is related to phase aberrations, which we assumed are located in the pupil plane (no instrumental scintillation). We only modeled low-order segment aberrations (piston, tip-tilt, defocus, astigmatism). Higher orders are less relevant for the optimization of the FPM size, but can have a significant impact on the coronagraphic performance.

The amplitude diffraction effect of gaps is partially accounted for (at least for infinitely small gaps) by the phase transition we are generating between primary mirror segments. 
Table 1. Optimum FPM diameter (and hence APLC characteristics) for several obscuration sizes and criteria.

\begin{tabular}{c|cc|cc}
\hline \hline & \multicolumn{2}{|c|}{$C_{\mathscr{C}}$} & \multicolumn{2}{c}{ Max. throughput } \\
Obstruction size (\%) & FPM $(\lambda / D)$ & Throughput $(\%)$ & FPM $(\lambda / D)$ & Throughput (\%) \\
\hline 10 & 4.3 & 59.4 & 4.1 & 62.2 \\
15 & 4.3 & 58.3 & 4.0 & 62.4 \\
20 & 4.4 & 55.8 & 3.8 & 65.5 \\
25 & 4.6 & 52.7 & 3.6 & 67.9 \\
30 & 4.7 & 51.2 & 3.5 & 68.7 \\
35 & 4.9 & 49.4 & 3.3 & 70.4 \\
\hline
\end{tabular}

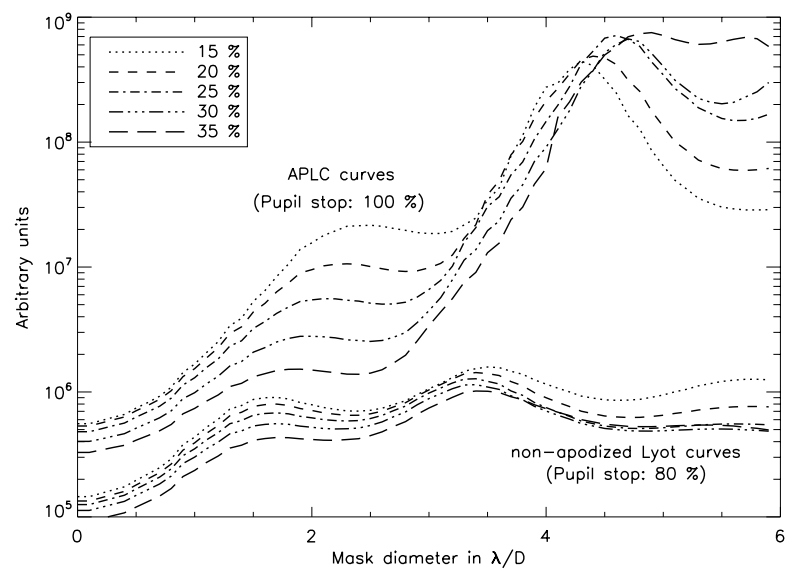

Fig. 4. $C_{\mathscr{C}}$ average between 3 and $100 \lambda / D$ as a function of the FPM diameter and obscuration sizes, in the case of the APLC and classical Lyot coronagraph.

\subsubsection{Central obscuration}

The first parameter we evaluate is the central obscuration. High contrast instruments have to deal with central obscuration ratios which typically range from $10 \%$ to $35 \%$ (CFHT: $35 \%$, HST: $33 \%$, VLT: $14 \%$ ). ELTs will likely have larger obscurations than current 8-m class telescopes to preserve a reasonable size for the telescope structure. In Fig. 4 , the criterion $C_{\mathscr{C}}$ is shown for different obscuration sizes ranging from 10 to $35 \%$. The curves show two maxima. The first is located near $2 \lambda / \mathrm{D}$ and experiences a large contrast variation while the second (near $4 \lambda / \mathrm{D}$ ) shows a smaller dispersion.

Table 1 summarizes these results and gives the position of the second maximum versus the obscuration size for the previouslymentioned criterion and for a criterion based solely on the maximum throughput (as in Fig. 3).

If we only consider the second maximum, which is more promising in terms of contrast and appears less sensitive, the optimal FPM diameter ranges from 4.3 to $4.9 \lambda / D$ for obscuration ratios between 10 to $35 \%$. Here, our criterion $C_{\mathscr{C}}$ is more relevant than throughput, since it is better adapted to the region of interest in the coronagraphic image and to the modification of the PSF structure. We see a non-linear increase of optimum FPM size with the obscuration ratio because more starlight is redistributed in the Airy rings of the PSF. A solely throughput-based consideration shows the opposite behavior with a larger dispersion of the FPM size, which is not consistent with the effect on the PSF structure. However, at small obscuration sizes $(10 \%-15 \%)$, maximum throughput yields a similar optimal FPM diameter as $C_{\mathscr{C}}$. We consider this result to be evidence for the relevance of our criterion $C_{\mathscr{C}}$ to optimize the FPM size (and hence the APLC characteristics) with respect to the size of the central obscuration. Moreover, the validity of our

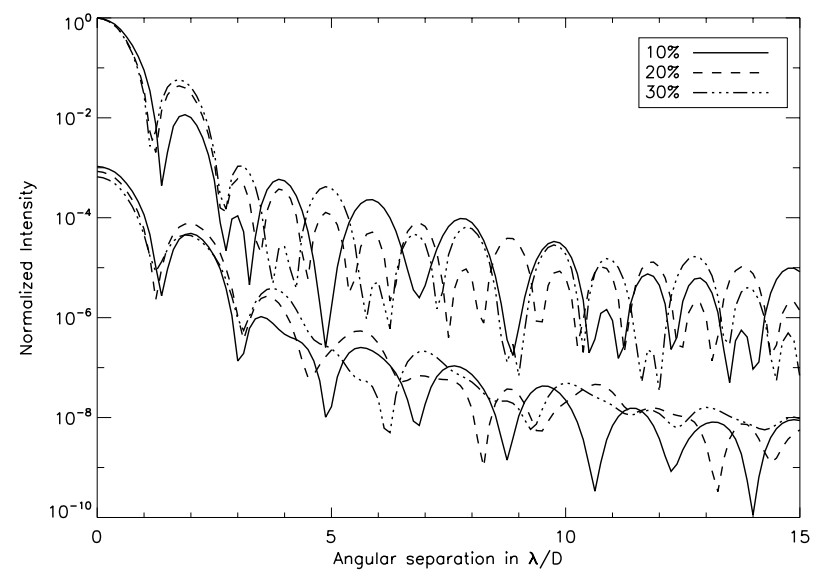

Fig. 5. Radial profiles of PSFs and coronagraphic images obtained with optimal APLC (using $C_{\mathscr{C}}$ ) for several obscuration sizes.

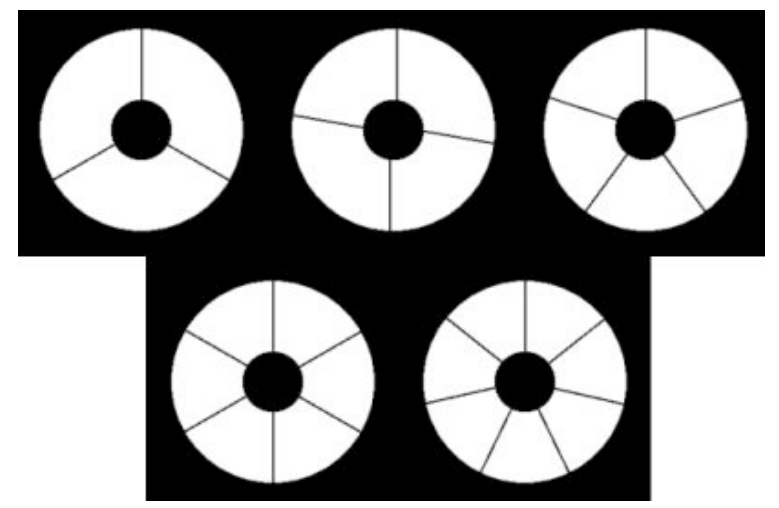

Fig. 6. Pupil configurations considered in this paper.

criterion is also supported by the comparison of coronagraphic PSFs using an optimized APLC in Fig. 5. The optimized APLC allows for a contrast performance which is rather insensitive to the central obscuration size.

\subsubsection{Spider arms}

On an ELT, the secondary mirror has to be supported by a complex system of spider arms $(\sim 50 \mathrm{~cm})$ and cables $(\sim 30-60 \mathrm{~mm})$ to improve stiffness. Evaluating the influence of these supports is important in the context of coronagraphy.

The pixel sampling of our simulations limited by available computer power does not allow us to model the thinnest mechanical supports. However, the impact of these supports on the PSF structure will be similar to that of spider arms but at a reduced intensity level. Several configurations were considered as shown in Fig. 6. As the number of spider arms increases from 3 to 7 , the contrast worsens (but no more than by a factor of 2). 


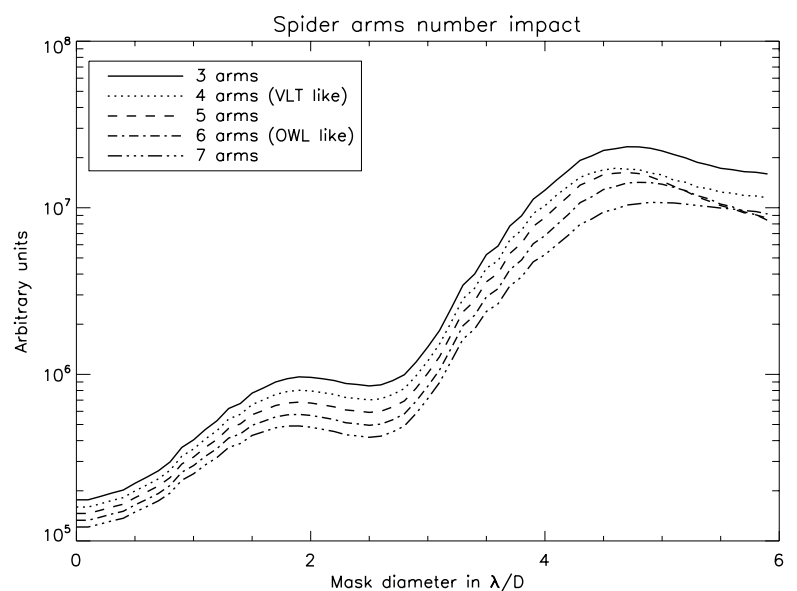

Fig. 7. $C_{\mathscr{C}}$ average between 3 and $100 \lambda / D$ as a function of the FPM diameter and number of spider arms. Spider thickness is set to $62 \mathrm{~cm}$.

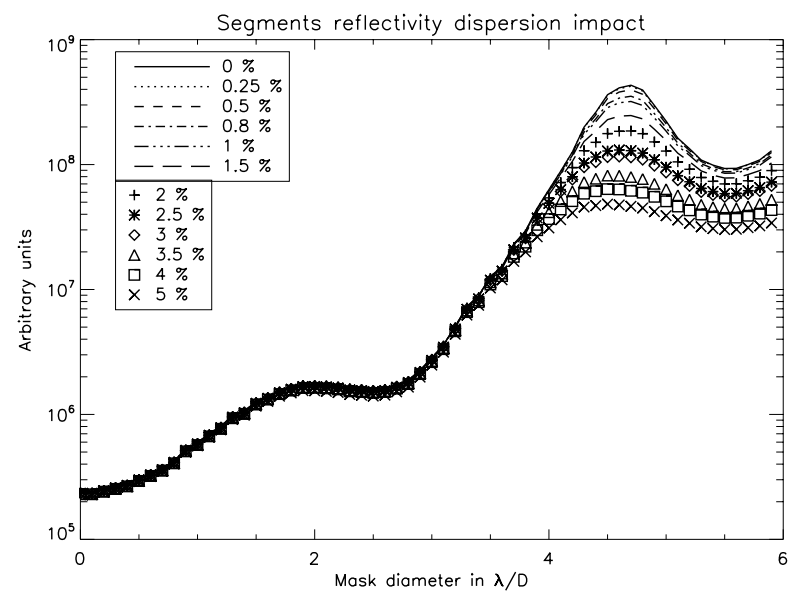

Fig. 8. $C_{\mathscr{C}}$ average between 3 and $100 \lambda / D$ as a function of the FPM diameter and reflectivity variations.

The curves in Fig. 7 are almost parallel, indicating that the number of spider arms has no significant influence on the optimal FPM size. The second maximum of $C_{\mathscr{C}}$ peaks at $4.7 \lambda / D$ with a small dispersion of $0.2 \lambda / D$.

Assuming a 6-spider arms configuration (OWL-like), we also analyzed the sensitivity to spider arm thickness from $15 \mathrm{~cm}$ to $93 \mathrm{~cm}$ (Fig. 9). The increasing width of the spider arms tends to flatten the profile of $C_{\mathscr{C}}$, making the selection of an optimal FPM more difficult (or less relevant) for very large spider arms. However, for the actual size of spider arms likely being of the order of $50 \mathrm{~cm}$, the optimal size of the FPM (and hence APLC) is still $4.7 \lambda / D$.

\subsubsection{Segments reflectivity variation}

The primary mirror of an ELT will be segmented because of its size, and a potential resulting amplitude effect is segmentto-segment reflectivity variation. We show the APLC optimization sensitivity for segment reflectivity variation from 0 to $5 \%$ peak-to-valley in Fig. 8. For this simulation, the primary mirror was assumed to consist of $\sim 750$ hexagonal segments. The criterion $C_{\mathscr{C}}$ is robust for FPMs smaller than $4 \lambda / D$. A loss of performance with reflectivity variation is observed for larger FPM. However, the optimal FPM size remains located at 4.7 $\lambda / D$ with a small dispersion of $0.2 \lambda / D$.

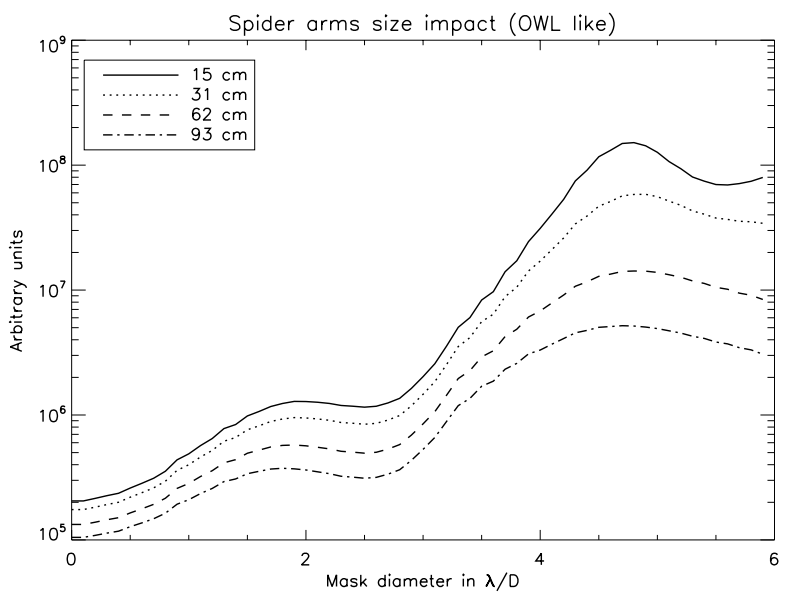

Fig. 9. $C_{\mathscr{C}}$ average between 3 and $100 \lambda / D$ as a function of the FPM diameter and spider arm thickness. Number of spider arms is set to 6 .

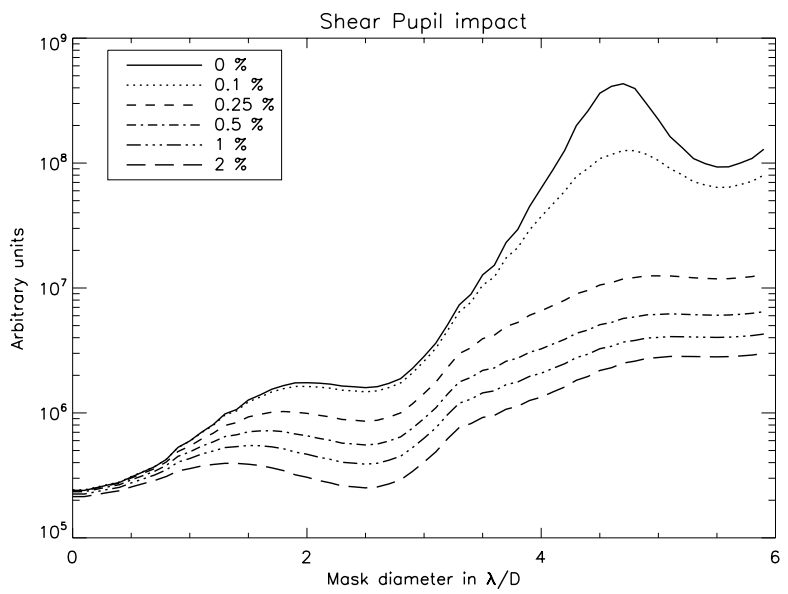

Fig. 10. $C_{\mathscr{C}}$ average between 3 and $100 \lambda / D$ as a function of the FPM diameter and pupil shear.

\subsubsection{Pupil shear}

As mentioned above, an APLC includes several optical components: apodizer, FPM and pupil stop. The performance of the APLC also depends on the alignment of these components. In particular, the pupil stop has to accurately match the telescope pupil image. This condition is not always satisfied, and the telescope pupil may undergo significant mismatch which could amount to more than $1 \%$ of its diameter. The pupil shear is the mis-alignment of the pupil stop with respect to the telescope pupil image. It is an issue especially for ELTs for which mechanical constraints are important for the design. For example, the James Webb Space Telescope is expected to deliver a pupil image for which the position is known at about $3-4 \%$. Therefore, the performance of the mid-IR coronagraph (Boccaletti et al. 2004) will be strongly affected. On SPHERE, the planet-finder instrument for the VLT (2010), the pupil shear was identified as a major issue and a dedicated Tip-Tilt mirror was included in the design to preserve the alignment at a level of $0.2 \%$ (Beuzit et al. 2006b).

The behavior of $C_{\mathscr{C}}$ in Fig. 10 is somewhat different from that seen with the previous parameters. The loss of performance is significant even for small FPM. However, the criterion is still peaking at $4.7 \lambda / D$ with a variation of about $0.2 \lambda / D$ although above $4.5 \lambda / D$ the curves are rather flat indicating that a larger FPM would not improve performance. 
Table 2. APLC optimization for an obscuration of $30 \%$.

\begin{tabular}{lcc}
\hline \hline Parameters & Value range & Optimal APLC configuration (FPM range in $\lambda / D$ ) \\
\hline Obscuration & $30 \%$ & 4.7 \\
Spider (arm) & $3-7$ & $4.6-4.8$ \\
Spider (size) & $15-90 \mathrm{~cm}$ & $4.6-4.8$ \\
Shear pupil & $0.5-2 \%$ & $4.7-4.9$ \\
Segment reflectivity & $0.25-5 \%$ & $4.5-4.7$ \\
Low-order aberrations & $1-100 \mathrm{~nm}$ rms & $3.5-6.0$ \\
Chromatism $(\Delta \lambda / \lambda)$ & $1.4-5 \%$ & $4.7-4.8$ \\
Chromatism $(\Delta \lambda / \lambda)$ & $5-20 \%$ & $4.8-5.3$ \\
\hline
\end{tabular}

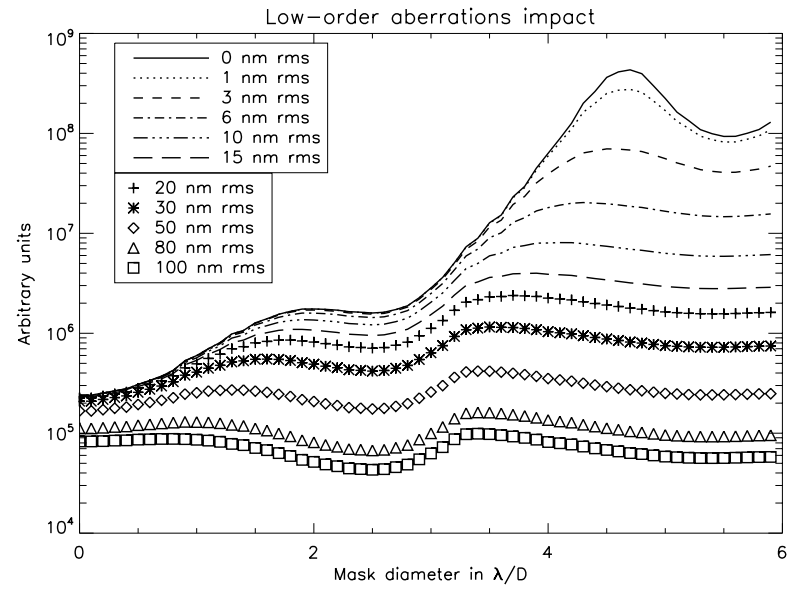

Fig. 11. $C_{\mathscr{C}}$ average between 3 and $100 \lambda / D$ as a function of the FPM diameter and low-order aberrations.

\subsubsection{Static aberrations}

Here, static aberrations refer to low-order aberrations on the segments of the large primary mirror. We separately investigated the effect of piston, tip-tilt, defocus and astigmatism, and found the behavior to be similar for all these aberrations. In contrast to the other defects, both the performance and the optimal FPM diameter (optimal APLC) are very sensitive to low-order aberrations.

As the amplitude of aberrations increases, the dependency of $C_{\mathscr{C}}$ on FPM diameter becomes flatter and the optimal FPM size gets smaller (Fig. 11). A larger FPM would not decrease performance enormously. For values larger than $15 \mathrm{~nm}$, there is no longer clear evidence of an optimal size beyond $\sim 3.5 \lambda / D$. The performance is rather insensitive to the actual FPM size.

Even though low-order aberrations strongly affect APLC performance, their presence has virtually no impact on the optimized configuration. The fairly constant performance in the presence of larger low-order aberrations indicates that low-order aberrations are not a relevant parameter for the optimization of the APLC.

\subsubsection{Chromatism}

All previous analysis was performed for monochromatic light of the wavelength $\lambda_{0}$. However, as with the classical Lyot coronagraph, the APLC performance should depend on the ratio between FPM size and PSF size and therefore on wavelength. Hence, the impact of chromatism on the APLC optimization must be evaluated. We note that the chromatism of the APLC can also be mitigated by a slight modification of the standard design (Aime 2005).

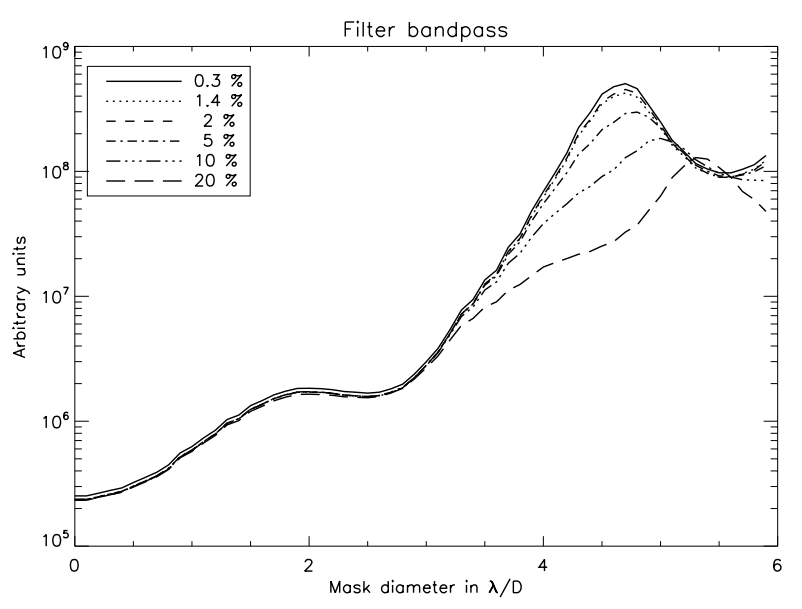

Fig. 12. $C_{\mathscr{C}}$ average between 3 and $100 \lambda / D$ as a function of FPM diameter and the filter bandpass.

Table 3. Chromatism effects synthesis.

\begin{tabular}{ccccc}
\hline \hline$\Delta \lambda / \lambda(\%)$ & $F P M(\lambda / D)$ & $F P M_{\lambda_{\max }}(\lambda / D)$ & $F_{1}$ & $F_{2}$ \\
\hline 0.3 & 4.70 & 4.70 & 1.0 & 1.0 \\
1.4 & 4.70 & 4.73 & 1.1 & 1.1 \\
2 & 4.70 & 4.75 & 1.1 & 1.1 \\
5 & 4.80 & 4.82 & 1.6 & 1.6 \\
10 & 5.00 & 4.94 & 2.6 & 3.7 \\
20 & 5.30 & 5.20 & 3.7 & 14.6 \\
50 & 5.90 & 5.87 & 26.3 & 180.9 \\
\hline
\end{tabular}

Figure 12 and Table 3 present the results of the simulations for several filter bandpass widths $(\Delta \lambda / \lambda)$ when using the standard monochromatic APLC. As long as the filter bandpass is smaller than 5\%, the optimal FPM size and performance are nearly the same as in the monochromatic case.

The values displayed in Cols. 4 and 5 of Table 3 quantify the loss of contrast due to chromaticity with respect to the monochromatic case for the APLC being optimized to the filter bandpass $\left(F_{1}\right)$ and to the central wavelength of the band $\left(F_{2}\right)$. These two factors begin to differ significantly from each other at a filter bandpass larger than 5\%. Hence, optimization of the APLC for chromatism is needed for a filter bandpass exceeding this value.

An efficient way of optimizing an APLC for broad band application is to optimize it for the longest wavelength of the band, which leads to results that are within $0.1 \lambda / D$ of the true optimal FPM size. This behavior can be explained by the nonsymmetrical evolution of the residual energy in the coronagraphic image around the optimal FPM size at $\lambda_{0}$ (Soummer et al. 2003). Another way to minimize chromaticity would be to calculate the apodizer profile for the central wavelength and only 

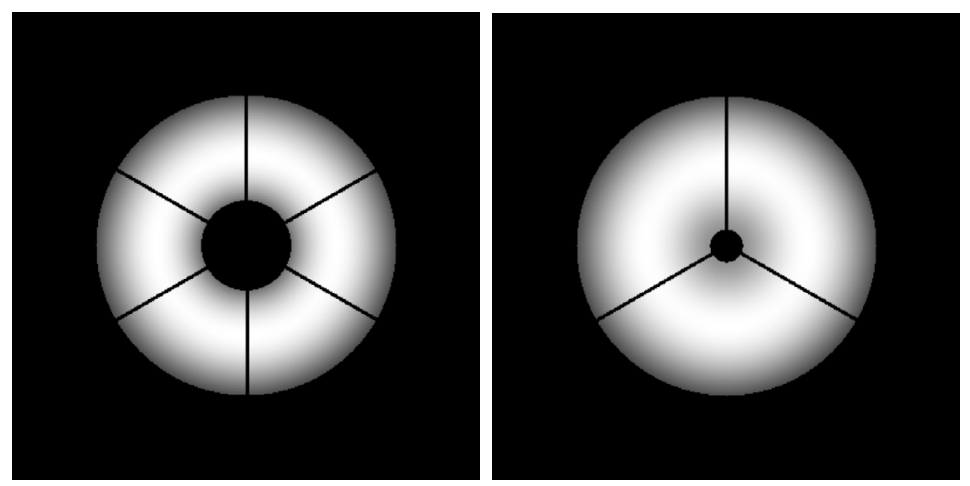

Fig. 13. Optimized apodized E-ELT apertures: telescope design 1 (left), telescope design 2 (right).
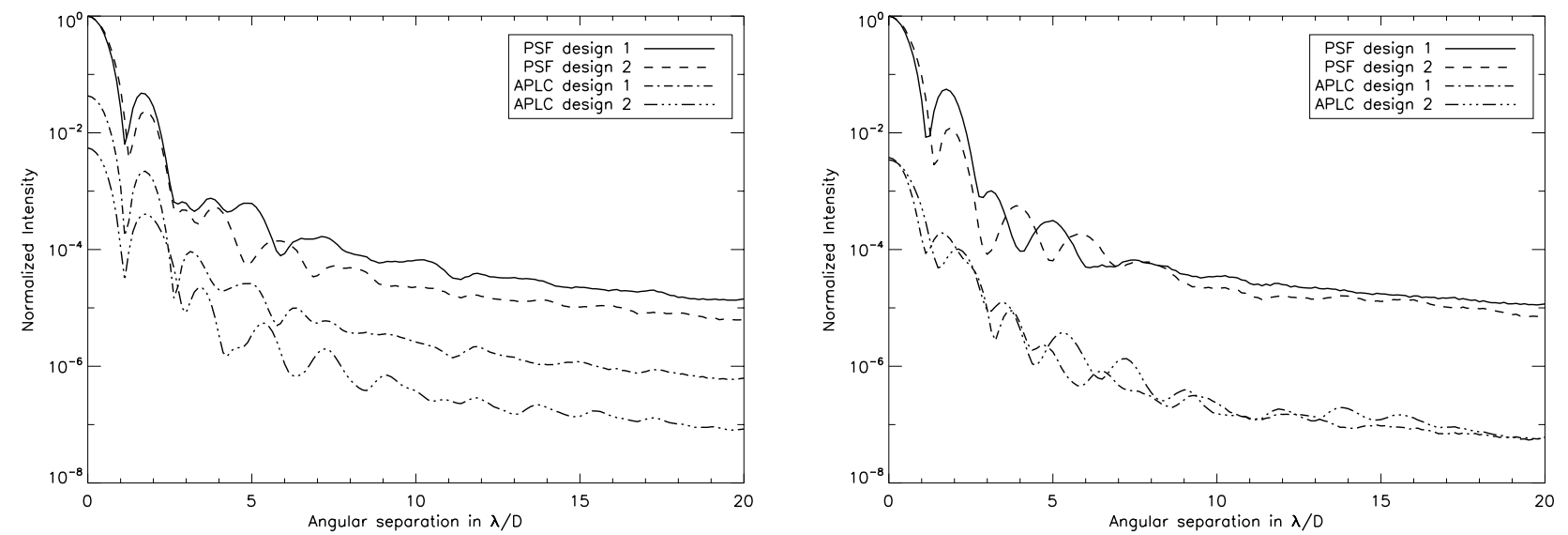

Fig. 14. Radial profiles of PSFs and coronagraphic images $(\Delta \lambda / \lambda=20 \%)$ for the 2 designs considering throughput optimization (up) or $C_{\mathscr{C}}$ optimization (bottom).

optimize the FPM diameter considering the whole bandpass. We compared the behavior of both methods for $\Delta \lambda / \lambda=20 \%$ : they are in fact very comparable in terms of performance.

\section{Application to the E-ELT}

In this section, we apply the tools and results from the APLC optimization study discussed in the previous section to the two telescope designs proposed for the E-ELT. The objective is to confirm our optimization method and to produce contrast idealized profiles which admittedly must not be confused with the final achievable contrast in the presence of a realistic set or instrumental error terms.

\subsection{Starting with telescope designs}

We assume a circular monolithic primary mirror of $42 \mathrm{~m}$ diameter. Segmentation errors are not taken into account, although we note that the E-ELT primary mirror consists of hexagonal segments with diameters ranging from 1.2 to $1.6 \mathrm{~m}$ in its current design.

Two competing telescope designs are considered: a 5-mirror arrangement (design 1) and a 2-mirror Gregorian (design 2). For our purpose, the two designs differ by their central obscuration ratios and the number of spider arms. Design 1 (Fig. 13 left) is a $30 \%$ obscured aperture with 6 spider arms of $50 \mathrm{~cm}$ and design 2 (Fig. 13 right) is a $11 \%$ obscured aperture with 3 spider arms of $50 \mathrm{~cm}$. These numbers are likely to be subject to change as the telescope design study is progressing. Mechanical supports (non-radial cables of the secondary mirror support) and intersegment gaps are not considered for the reasons mentioned in Sect. 3.2.2.

In such conditions and taking into account the previous sensitivity analysis on central obscuration, spider arms, and chromatism $(\Delta \lambda / \lambda=20 \%)$ we found an optimal APLC configuration with the apodizer designed for 4.8 and $4.3 \lambda / D$ and with a FPM size of 5 and $4.3 \lambda / D$ for design 1 and 2, respectively.

Sivaramakrishnan \& Lloyd (2005) has demonstrated that optimization or under-sizing of the pupil stop is not necessary with the APLC. We independently verified and confirm this result using our criterion applied to the stop rather than to the mask.

\subsection{Radial contrast}

As shown in Sect. 3.2.1, the optimal APLC configuration with our criterion is different to the optimal configuration considering throughput as a metric. We can now demonstrate this difference using contrast profiles. Figure 14 compares the coronagraphic profiles based on throughput optimization (apodizer and FPM size are 3.5 and $4.1 \lambda / D$ for design 1 and 2 , see Fig. 3 ) with that obtained from optimization with our criterion.

For design 2, the optimization with both methods leads to similar APLC configurations (4.3 and $4.1 \lambda / D)$. Hence, the contrast performance between them differs by only a factor of 3 . On the other hand for design 1, the gain by using our criterion for the optimization is a staggering factor 10 . In addition, the plot shows that APLC contrast performance only weakly depends on the telescope geometry with this optimization method. This is an important result, which means that the APLC can efficiently cope with a large variety of telescope designs. 


\section{Conclusion}

The APLC is believed to be a well suited coronagraph for ELTs and for the search of extrasolar planets with direct imaging. The high angular resolution of such large telescopes relaxes the constraints on the IWA of a coronagraph which is an important issue for high contrast imaging instruments on 8-m class telescopes. Hence, coronagraphs with a relatively large IWA such as the APLC present an interesting alternative to the small IWA coronagraphs such as the phase mask coronagraphs.

The objective of this paper is to analyze the optimization of APLC in the context of ELTs. We defined a criterion $\left(C_{\mathscr{C}}\right)$ similar to that used by Boccaletti (2004) for the general problem of Lyot stop optimization in coronagraphy. We then analyzed the behavior of this criterion as a function of the FPM diameter in the presence of different telescope parameters. The optimal FPM is determined by the maximum value of the criterion. A sensitivity analysis was carried out for the several telescope parameters such as central obscuration, spiders, segment reflectivity, pupil shear, low-order static aberrations and chromatism. Some of these parameters are not relevant for APLC optimization such as low-order aberrations which provide a pretty flat response of the criterion to FPM diameter when applied at reasonably large amplitudes. However, ELTs are not yet well enough defined to predict the level of static aberrations coronagraphs will have to deal with.

The parameter which had the largest impact on the optimum FPM diameter is the central obscuration. An obscuration ratio of $30 \%$ leads to an optimal APLC of $4.7 \lambda / D$. In most cases, the optimal sizes derived for other telescope parameters are quite consistent with that imposed by the central obscuration. The dispersion of the FPM size is no larger than $0.2 \lambda / \mathrm{D}$ given the range of parameters we have considered. We also demonstrated that APLC optimization based on throughput alone is not appropriate and leads to optimal FPM sizes which decrease with increasing obscuration ratios. This behavior is opposite to that derived using our criterion. The superior quality of our criterion is supported by the comparison of contrast profiles obtained with both optimization methods in Sects. 4.2 and 3.2.1.

Although the idealized simulations presented in this paper do not consider atmospheric turbulence and instrumental defects, they allow us to find the optimal APLC configuration and PSF contrast for each case. Cavarroc et al. (2006) show that the ultimate contrast achievable by differential imaging (speckle noise suppression system to enhance the contrast, Racine et al. 1999; Marois et al. 2000; Baba \& Murakami 2003; Guyon 2004) with a perfect coronagraph is not sensitive to atmospheric seeing but depends critically on static phase and amplitude aberrations. Our results therefore present the possibility of extending this study to the more realistic case of a real coronagraph taking into account relevant effects releated to telescope properties.

In addition, we have also started development of APLC prototypes whose characteristics are defined with the present numerical analysis. Experiments with these prototypes will be carried out during the next year in the near IR on the High Order Test-bench (Vernet et al. 2006) developed at the European Southern Observatory. The practical study of the APLC will also benefit from prototyping activities led by the department of Astrophysics at the University of Nice (LUAN) and carried out for development of SPHERE for the VLT.

Acknowledgements. P.M. would thanks Pierre Riaud for helpful discussions. This activity is supported by the European Community under its Framework Programme 6, ELT Design Study, Contract No. 011863.

\section{References}

Aime, C. 2005, PASP, 117, 1012

Aime, C., Soummer, R., \& Ferrari, A. 2002, A\&A, 389, 334

Andersen, T., Ardeberg, A. L., Beckers, J., et al. 2003, in Future Giant Telescopes, Presented at the Society of Photo-Optical Instrumentation Engineers, ed. J. R. P. Angel, \& R. Gilmozzi, Proc. SPIE Conf., 4840, 214 Baba, N., \& Murakami, N. 2003, PASP, 115, 1363

Beuzit, J.-L., Feldt, M., Dohlen, K., et al. 2006a, The Messenger, 125, 29

Beuzit, J. L., Mouillet, D., Moutou, C., et al. 2006b, in Tenth Anniversary of 51 Peg-b: Status of and prospects for hot Jupiter studies, ed. L. Arnold, F. Bouchy, \& C. Moutou, 353

Boccaletti, A. 2004, in EAS Publications Series, ed. C. Aime, \& R. Soummer, 165

Boccaletti, A., Riaud, P., Baudoz, P., et al. 2004, in EAS Publications Series, ed. C. Aime, \& R. Soummer, 195

Cavarroc, C., Boccaletti, A., Baudoz, P., Fusco, T., \& Rouan, D. 2006, A\&A, 447, 397

Dierickx, P., Brunetto, E. T., Comeron, F., et al. 2004, in Ground-based Telescopes, Presented at the Society of Photo-Optical Instrumentation Engineers, ed. J. M. Oschmann, Jr., Proc. SPIE Conf., 5489, 391

Gerchberg, R. W., \& Saxton, W. O. 1972, in Optik 35, 237

Guyon, O. 2004, ApJ, 615, 562

Johns, M., Angel, J. R. P., Shectman, S., et al. 2004, in Ground-based Telescopes, Presented at the Society of Photo-Optical Instrumentation Engineers, ed. J. M. Oschmann, Jr., Proc. SPIE Conf., 5489, 441

Kuchner, M. J., \& Traub, W. A. 2002, ApJ, 570, 900

Macintosh, B., Graham, J., Palmer, D., et al. 2006, in Advances in Adaptive Optics II, Presented at the Society of Photo-Optical Instrumentation Engineers, ed. B. L. Ellerbroek, \& D. Bonaccini Calia, Proc. SPIE Conf., $6272,62720 \mathrm{~L}$

Marois, C., Doyon, R., Racine, R., \& Nadeau, D. 2000, PASP, 112, 91

Mawet, D., Riaud, P., Absil, O., \& Surdej, J. 2005, ApJ, 633, 1191

Nelson, J., \& Sanders, G. H. 2006, in Ground-based and Airborne Telescopes, Presented at the Society of Photo-Optical Instrumentation Engineers, ed. L. M. Stepp, Proc. SPIE Conf., 6267, 626728

Racine, R., Walker, G. A. H., Nadeau, D., Doyon, R., \& Marois, C. 1999, PASP, 111,587

Roddier, F., \& Roddier, C. 1997, PASP, 109, 815

Rouan, D., Riaud, P., Boccaletti, A., Clénet, Y., \& Labeyrie, A. 2000, PASP, 112, 1479

Sivaramakrishnan, A., \& Lloyd, J. P. 2005, ApJ, 633, 528

Soummer, R. 2005, ApJ, 618, L161

Soummer, R., Aime, C., \& Falloon, P. E. 2003, A\&A, 397, 1161

Soummer, R., Pueyo, L., Ferrari, A., et al. 2007, ApJ, submitted

Vernet, E., Kasper, M., Vérinaud, C., et al. 2006, in Advances in Adaptive Optics II, ed. B. L. Ellerbroek, \& D. Bonaccini Calia, Proc. SPIE, 6272, $62722 \mathrm{~K}$ 